\title{
Endoscopic ultrasound-guided needle-based confocal laser endomicroscopy for diagnosis of solid pancreatic lesions (ENES): a pilot study
}

Authors

Institutions
Pradermchai Kongkam', Rapat Pittayanon ${ }^{1}$, Pichet Sampatanukul'2, Phonthep Angsuwatcharakon ${ }^{1}$, Satimai Aniwan', Piyapan Prueksapanich', Virote Sriuranpong', Patpong Navicharern ${ }^{3}$, Sombat Treeprasertsuk', Pinit Kullavanijaya', Rungsun Rerknimitr ${ }^{1}$

Institutions are listed at the end of article. submitted 13. April 2015 accepted after revision 17. August 2015

\section{Bibliography}

Dol http://dx.doi.org/ 10.1055/s-0034-1393183

Published online: 5.11.2015

Endoscopy International Open 2016; 04: E17-E23

(c) Georg Thieme Verlag KG Stuttgart · New York

E-ISSN 2196-9736

Corresponding author Pradermchai Kongkam, MD Gastrointestinal Endoscopy Excellence Center

Department of Medicine Faculty of Medicine Chulalongkorn University and King Chulalongkorn Memorial Hospital

Thai Red Cross Society

1873, Rama 4 Road

Patumwan

Bangkok

Thailand 10500

Fax: +66-2-6524219

kongkam@hotmail.com
Background and study aims: Endoscopic ultrasound-guided needle-based confocal laser endomicroscopy (EUS-nCLE) has been shown to aid in the diagnosis of cystic pancreatic lesions. This is a pilot project to study its findings in patients with solid pancreatic lesions (SPLs) with a prospective single-blinded study design.

Methods: Patients with SPLs undergoing transgastric EUS fine needle aspiration (EUS-FNA) from July 2013 to March 2014 were prospectively enrolled. The nCLE diagnoses were compared with the final diagnoses. Researchers learned about the EUS-nCLE findings from previously published studies and applied it to diagnose SPLs. In the meantime, the findings were recorded.

Results: In total, 22 patients were recruited (mean age 62.7 years, SD 13.8 years; 14 men and eight women). The mean maximal tumor diameter was $36.0 \mathrm{~mm}$ (SD $10.9 \mathrm{~mm}$ ). EUS-nCLE yielded satisfactory images in all patients during the first EUS procedure and diagnosed benign and malignant SPLs in 3 and 19 patients, respectively. Final diagnoses of malignant SPLs were made in 19 patients. Benign SPLs were eventually diagnosed in

\section{Introduction}

$\nabla$

Preoperative diagnosis of solid pancreatic lesions (SPLs) is crucial. Erroneous diagnosis may lead to unnecessary surgery for benign SPLs or may result in the progression of early stage pancreatic cancer to an incurable one. Endoscopic ultrasound-guided fine needle aspiration (EUS-FNA) is currently considered to be the best test for diagnosing SPLs, with reported sensitivity and specificity rates of $91 \%$ and $94 \%$, respectively $[1,2]$. However, EUS-FNA has certain limitations, including requiring an onsite cytopathologist to enhance the efficacy of the procedure, which is a limitation at many institutions [3]. three patients, with confirmed the cytology and disease stability during the 12-month follow-up period. At the end of the project, based on the results of this current study, EUS-nCLE findings for malignant SPLs were dark clumping with or without dilated vessels $(>40 \mu \mathrm{m})$. There were two criteria for diagnosing benign lesions which were white fibrous bands and normal acini cells. The accuracy rate of EUS-nCLE was 90.9\% (20/22). One falsely diagnosed malignant SPL was an inflammatory mass from a recent acute pancreatitis. Another one with a pancreatic neuroendocrine tumor presenting with a symptomatic pseudocyst was incorrectly diagnosed as an inflammatory mass. This was likely from sampling error of the EUS-nCLE probe in an inflammatory area. Only one patient had post EUS-FNA bleeding but did not require a blood transfusion. The inter-observer agreement among three blinded endoscopists was almost perfect (Kappa 0.82).

Conclusion: EUS-nCLE is a promising technique for the diagnosis of SPLs with good inter-observer agreement.

\section{Study registration: TCTR20140402001}

Needle-based confocal laser endomicroscopy (nCLE) is a novel imaging technology that can provide real-time magnified endoscopic images at the cellular level at 1000-fold magnification. It can be inserted through a 19-guage EUS-FNA needle. It was used in a few studies for diagnosing SPLs, nevertheless, no center has reported the results of its clinical application in a prospective fashion; we therefore prospectively assessed, in a blinded manner, the findings of EUS-nCLE in SPLs as a pilot study. In addition, we evaluated interobserver agreement in all off-line images. 


\section{Patients and methods}

$\nabla$

\section{Patients}

Only the multi-centered study conducted by Giovanini et al. has thus far reported the findings of EUS-nCLE in SPLs in abstract form [4]. We hence reviewed the abstract and video findings obtained from the study through http://www.cellvizio.net, and performed EUS-nCLE in known cases of pancreatic adenocarcinoma $(n=2)$ and mass-forming chronic pancreatitis $(n=1)$. Multiple reviews from these images made us sufficiently confident to interpret the results of EUS-nCLE from SPLS.

This investigation was performed as a prospective single-blinded study. It was conducted during the period July 2013 to March 2014, after permission had been granted from the institutional review board (IRB) of Chulalongkorn University, Bangkok, Thailand (IRB number 472/55, approval date February 7, 2013). The study was registered at www.clinicaltrials.in.th (TCTR identification number TCTR20140402001). The study was funded by King Chulalongkorn Memorial Hospital and Faculty of Medicine, Chulalongkorn University, Bangkok, Thailand. The inclusion criteria were as follows: patients with identified SPLs from prior computed tomography (CT) or magnetic resonance imaging (MRI); patients in whom EUS-nCLE could be performed transgastrically; patients with probable cancer masses considered potentially unresectable (in order to avoid the risk of seeding in potentially resectable ones); and patients older than 18 years. The reason that we performed only a transgastric procedure was because, at that time, we thought that the nCLE miniprobe could be easily damaged if a transduodenal approach was selected. The exclusion criteria included the presence of a potentially resectable cancer mass (as we were concerned about possible transgastric seeding from the EUS-FNA procedure); SPLs identified by previous radiological methods that were confirmed as cystic masses during the EUS procedure, allergy to fluorescein, renal insufficiency, and pregnancy or breast-feeding. The withdrawal criterion was technical failure of the EUS-nCLE procedure. An informed consent was obtained from every patient before the procedure.

\section{Methods}

\section{Statistics and sample size calculation}

As a prospective study of EUS-nCLE for diagnosing SPLs had never been conducted before this study, this current project was hence a pilot one. We planned to recruit 24 patients to accomplish the purpose of the study.

Cohen's kappa ( $\mathrm{K}$ ) was used to analyze inter-observer agreement. The values of kappa ( $\mathrm{K}$ ) for agreement were graded as slight agreement for 0.01 to 0.20 , fair for 0.21 to 0.40 , moderate for 0.41 to 0.60 , substantial for 0.61 to 0.80 , and almost perfect agreement for 0.81 to 1.00 .

\section{nCLE miniprobe}

The details of the nCLE miniprobe have been described previously [5]. Briefly, the nCLE miniprobe has a diameter of $0.85 \mathrm{~mm}$, has a $1000 \times$ magnification at the cellular level, and can be inserted through a 19-gauge EUS-FNA needle. To enhance mass imaging, $10 \%$ fluorescein sodium (range $2.5-5 \mathrm{~mL}$ ) was injected intravenously immediately before placing the EUS-FNA needle and nCLE miniprobe into the mass.

\section{Procedures}

Patient data, radiological imaging data, and relevant clinical information were reviewed and recorded by the research team. Patients who met the inclusion criteria and who had SPLs that could potentially be punctured by the transgastric EUS-FNA/EUS-nCLE procedure were brought to the endoscopy room. The procedure was performed using one of the linear-array echoendoscopes (EG-530UT2, FUJIFILM Corporation, Tokyo, Japan; GF-UCT140AL5, Olympus Medical System Corp., Japan).

EUS-FNA was performed with a blunt-tipped 19-gauge Access needle (EchoTip Ultra HD Ultrasound Access Needle; ECHO-HD19-A; Wilson-Cook Endoscopy, Winston-Salem, NC, USA). The EUS-FNA needle must be a 19-gauge needle to allow the nCLE probe to pass through it. Previously in our center, we experienced partial tears of the guidewire during EUS-guided therapeutic procedures when the wire is withdrawn back into the EUS-FNA needle. For this reason, to avoid possible damage to the nCLE miniprobe, we specifically chose the Access needle due to its blunt tip and selected only SPLs that could be approached transgastrically.

Before starting the procedure, the nCLE miniprobe was inserted into the EUS-FNA needle. The tip of the nCLE miniprobe was placed 3-5 mm outside the tip of the needle. The external end of the nCLE miniprobe was then secured with a locking device to maintain an accurate distance from the EUS-FNA needle sheet. Next, the miniprobe was removed and replaced with the stylet of the EUS-FNA needle, and the standard EUS-FNA procedure was performed. After the endosonographer had inserted the needle into the target area, the stylet was removed and replaced with the nCLE miniprobe using a post-loading technique as previously described [5]. Before the FNA needle was inserted into the target area, $2.5-5 \mathrm{~mL}$ of $10 \%$ fluorescein sodium was injected. The endosonographer (P.K.) adjusted the needle to the most appropriate area according to his clinical judgment. The interpreter (R.P.), who was blinded to the relevant clinical information was then brought into the room. The ultrasound images were kept away from her view. After she had identified the optimal images for making a SPL diagnosis, she subsequently informed the endosonographer to slow down or stop the movement of the EUS-FNA needle. She then read the nCLE images until she was satisfied with the results and later made a diagnosis based on these images. The nCLE diagnosis was classified as either a benign or a malignant SPL. If the interpreter could not identify satisfactory images, she informed the endosonographer to move the nCLE miniprobe to another location using the standard fanning technique (moving the nCLE miniprobe back and forth, up and down, or left and right inside the mass). If satisfactory images could not be obtained even after repositioning the nCLE miniprobe, the EUS-FNA needle and nCLE equipment were removed and the target area repunctured. During the re-adjustment procedure, the interpreter was asked to view only nCLE imaging. After the diagnosis had been made, the probe was removed from the sheet. Then, the endosonographer initiated the standard EUSFNA procedure and sampled the tissue from the target area. This tissue was sent to the cytopathologist (P. S.), who made a cytopathological diagnosis. Cytopathological diagnoses were classified into positive for malignancy, suspicious for malignancy, benign cells, and tissue inadequate for interpretation. 
Table 1 Classification of masses as definite or probable.

\begin{tabular}{|lll|}
\hline Level of diagnosis & Benign mass & Malignant mass \\
\hline Definite & Histology+stable at 12 months follow-up & Histology with or without disease progression within a 12-month follow-up period \\
\hline Probable & Stable at 12 months follow-up alone & Follow-up within or at 12 months shows progression \\
\hline
\end{tabular}

The numbers of criteria were different between the benign and the malignant solid pancreatic lesion, as benign tissue cannot be definitively diagnosed. Adequate follow-up time is therefore required to confirm a definite diagnosis of benign solid pancreatic lesions.

\section{EUS-nCLE findings and diagnosis}

During the study period, after finishing each EUS-nCLE procedure and after diagnosis was made in each patient, our interpreter of EUS-nCLE findings (R.P.) was informed about all clinical information and the most likely diagnoses. Certainly, the final diagnoses, once available, were reported to her as well. This was in order to improve her diagnostic skill as a learning curve. As the study went by, case by case, she learned, created, and adapted EUS-nCLE findings to aid the diagnosis of SPLS. However, EUSnCLE diagnoses to be compared with final diagnoses were obtained during the procedure by our interpreter (R.P.).

\section{Inter-observer agreement}

All nCLE images were collected at the end of the study and presented in off-line mode to three experienced endoscopists (P. K., R. P., and P. P.) who were blinded to all clinical and other related information to make a diagnosis of SPLs as either benign or malignant ones. Results were calculated for inter-observer agreement.

\section{Final diagnoses}

The nCLE diagnoses were compared with the final diagnoses, the latter of which were considered to be the gold standard. Final diagnoses were classified into two groups: definitive and probable diagnoses ( $\bullet$ Table1). For benign SPLs, the criteria for a definitive diagnosis were (1) confirmed histology (by FNA or histology) and (2) stable disease after a 12-month follow-up period. Alternatively, a probable diagnosis was made when only the second criterion was met. For malignant SPLs, the criterion for a diagnosis was a pathological diagnosis; a probable diagnosis was assigned when the 12-month follow-up exam revealed progression of the disease or masses.

Finally, the nCLE diagnoses were compared with the final diagnoses. The sensitivity, specificity, positive predictive value, negative predictive value, and accuracy rate were calculated by SPSS program version 16.0. Clinical information, demographic data, and final diagnoses were compared between benign and malignant SPLS.

\section{Adverse events}

Before, during, and after the procedure, standard care for EUSFNA patients was applied. Any procedure-related adverse events experienced by the patients were recorded. Admission to the hospital was under the discretion of the responsible physician. Any mishaps involving the EUS equipment, accessories, nCLE machine, or needle were also recorded [6].

\section{Results}

\section{$\nabla$}

A total of 27 patients were eligible for the study. Two patients were excluded for the following reasons: the SPL was determined to be resectable during the EUS procedure (as we were concerned about possible transgastric seeding from the EUS-FNA procedure) $(n=1)$ and the SPL was found to be a solid-cystic mass during the EUS procedure $(n=1)$. Subsequently, 25 patients were recruited. Three patients were withdrawn from the study for the following reasons: the EUS-FNA needle and nCLE miniprobe could not reach the target area which was located in the head of the pancreas $(n=1)$; EUS-FNA could not be performed due to intervening collateral vessels $(n=1)$; and an inadequate specimen was obtained from EUS-FNA, and the patient denied follow-up $(\mathrm{n}=1)$. Eventually, the results from 22 patients (mean age 62.7 years, SD 13.8, 14 men/8 women) were analyzed. These patients presented with weight loss $(n=19)$, abdominal pain $(n=17)$, and jaundice $(n=11)$, in addition to one case of an incidental finding as shown in $\odot$ Fig.1.

Masses were primarily observed in the head $(n=15)$ of the pancreas, but the body $(n=6)$ and tail $(n=1)$ were also involved in certain cases. The mean maximal tumor diameter was $36.0 \mathrm{~mm}$ (SD $10.9 \mathrm{~mm}$ ). The median time for the EUS-nCLE procedures was 8.2 minutes (range $1-32$ minutes). The median number of

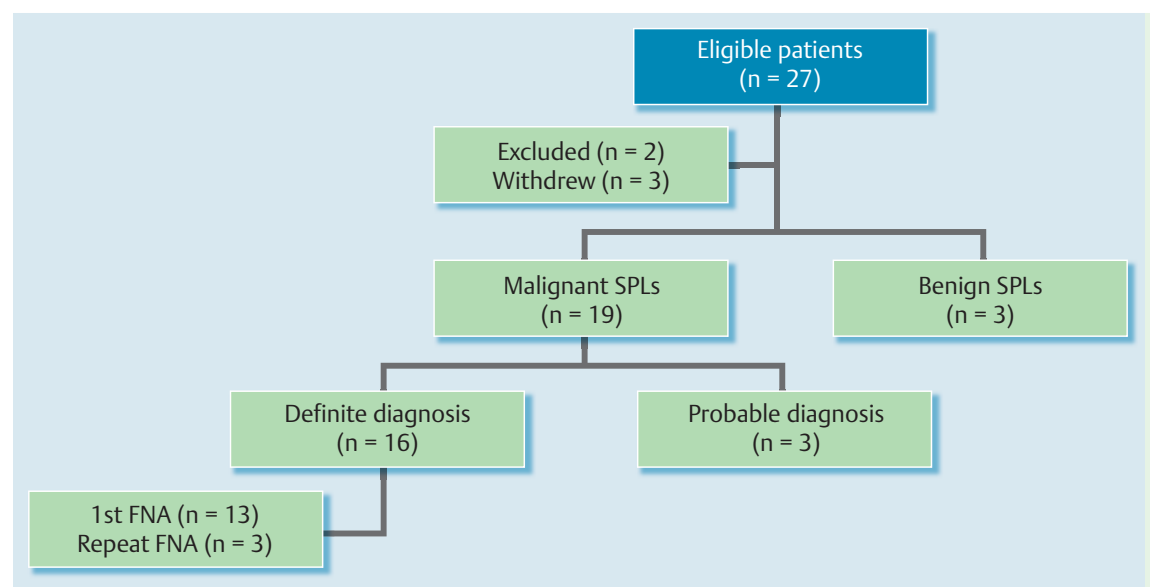

Fig. 1 Number of patients recruited into the study. In total, 27 patients with SPLs were eligible for the study. Two were excluded and three withdrew from the study. From 22 recruited patients, final diagnoses of malignant and benign SPLs were made in 19 and 3 patients, respectively. Among the 19 patients with malignant SPLs, 16 and 3 of them met definite and probable diagnostic criteria, respectively. In the 16 patients who met definite diagnostic criteria of malignant SPLs, 13 were diagnosed from positive results from the 1st EUS-FNA that was performed at the same time as the EUS-nCLE procedure. Abbreviations: SPLs, solid pancreatic lesions; EUS-nCLE, endoscopic ultrasound guided needle confocalbased endomicroscopy. 
Table 2 Endoscopic ultrasound-guided needle-based confocal laser endomicroscopy (EUS-nCLE) findings were classified according to the final diagnoses of benign and malignant SPLs.

\begin{tabular}{|llc|}
\hline Parameter/final diagnoses & Benign SPLs, $\mathbf{n}$ & Malignant SPLs, $\mathbf{n}$ \\
\hline Dark clumps $>40 \mu \mathrm{m}$ & 1 & 18 \\
\hline Dilated vessels & 0 & 5 \\
\hline Fine white fibrous bands & 3 & 0 \\
\hline Small black cell movement & 1 & 11 \\
\hline Normal acinar cells & 2 & 0 \\
\hline
\end{tabular}

SPLs, solid pancreatic lesions.

nCLE passages was 1 time (range 1 - 2 times). EUS-nCLE produced satisfactory images for all patients during the first EUS procedure. EUS-nCLE was diagnosed as benign and malignant SPLs in 3 and 19 patients, respectively. Nineteen patients were finally diagnosed as having malignant SPLs. Definite diagnoses of malignant SPLs were made in 16 patients based on the results of EUS-FNA. Thirteen of these 16 patients were diagnosed by the first FNA that was performed at the same time as the EUS-nCLE procedure. Another three patients were diagnosed as probable because either the 12-month follow-up showed progression of the disease $(n=1)$, or death from a pancreatic cancer-related cause occurred at 3 or 5 months $(n=2)$. Benign SPLs were eventually diagnosed in three patients, with confirmed cytology and with stable disease during the 12-month follow-up period in all patients.

- Table 2 shows the findings from EUS-nCLE in each group of patients. After the progression of experience from case-by-case learning during the study, the results of EUS-nCLE from SPLS were categorized as follows. Findings mainly obtained from malignant SPLs in this current study were dark clumps of cells larger than $40 \mu \mathrm{m}$ with or without dilated vessels larger than $20 \mu \mathrm{m}$, as shown in Fig. 2 and Video 1. For benign inflammatory lesions, fine white fibrous bands, as shown in Fig. 3 and - Video 2, were detected. Additionally, the findings observed in the normal pancreatic parenchyma were small black cell movements and normal acini.

The results of the EUS-nCLE were compared with the final diagnoses ( Table 3). Details with regard to the final diagnoses and

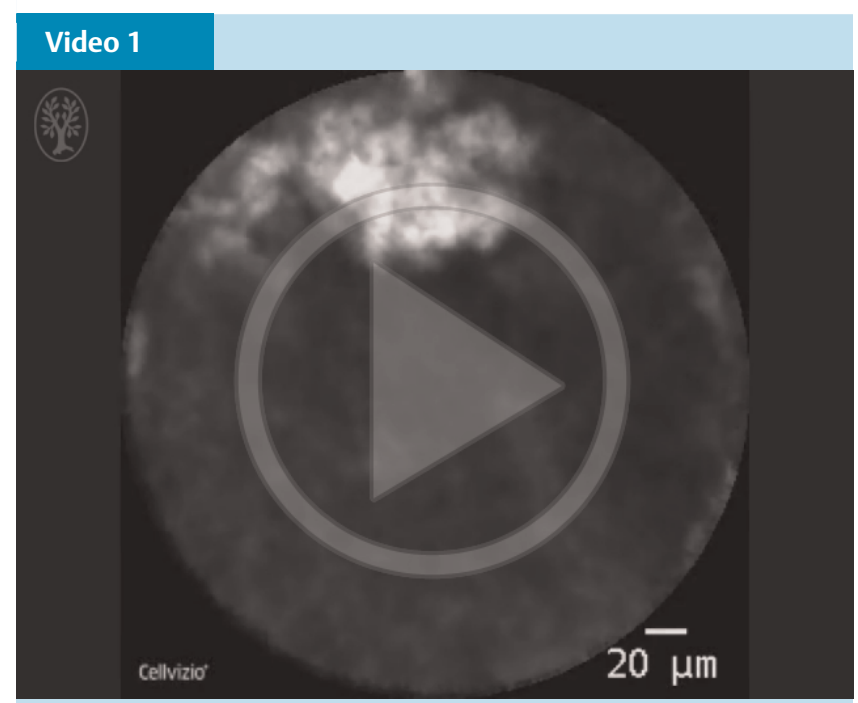

EUS-nCLE of pancreatic adenocarcinoma showing dark clumps of cells. Online content including video sequences viewable at: http://dx.doi.org/ 10.1055/s-0034-1393183

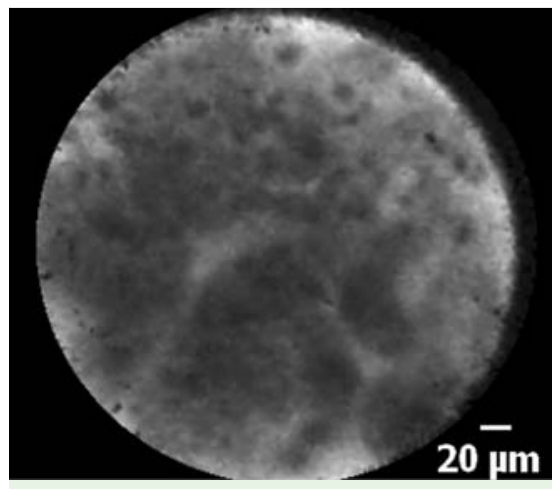

Fig.2 Image obtained from a patient with a final diagnosis of pancreatic adenocarcinoma.

This image was described as having dark clumps and was used as an nCLE sign of a malignant solid pancreatic lesion. We hypothesized that fluorescein could not enter these malignant cells; therefore, the black color of groups of cells can be observed in this image.

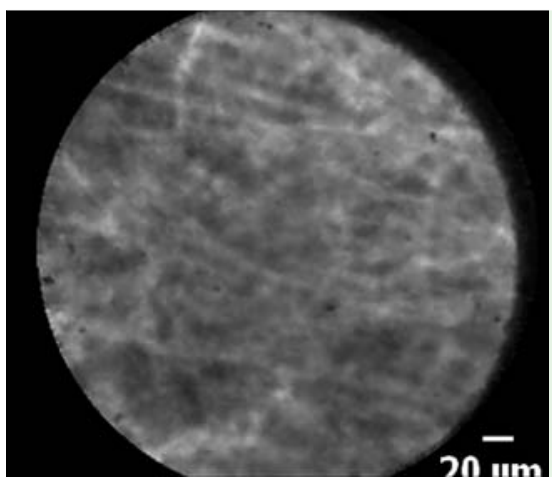

Fig.3 Image obtained from a patient with mass-forming chronic pancreatitis. This image was described as having fine white fibrous bands and was used as an nCLE sign of a non-malignant solid pancreatic lesion.

number of patients with each disease are described in $\square$ Table 4 and Table 5, respectively. Of those 19 malignant SPLs, findings from EUS-nCLE made a correct diagnosis in 18 (94.7\%). One falsely diagnosed malignant SPL was a lesion in a 56-year-old woman with recent acute focal pancreatitis who developed an inflammatory mass in the pancreas. After the EUS-nCLE and EUS-FNA had been performed, the mass eventually disappeared at 7 months after the EUS procedure. EUS-nCLE finding in this case was a dark clump of cells. In contrast, one pancreatic neuroendocrine tumor in a 43-year-old man who presented with abdominal

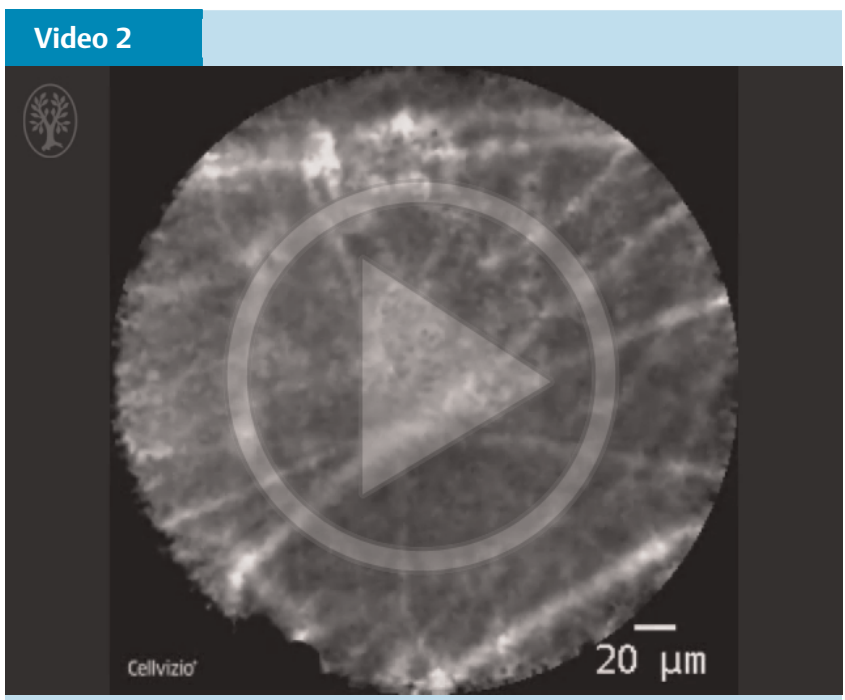

EUS-nCLE of benign inflammatory lesion showing fine white fibrous bands. Online content including video sequences viewable at: http://dx.doi.org/ $10.1055 / \mathrm{s}-0034-1393183$ 


\begin{tabular}{|lllc|}
\hline Diagnoses & $\begin{array}{l}\text { Benign SPLs }(\mathbf{n}=\mathbf{3}) \\
\text { (final diagnosis) }\end{array}$ & $\begin{array}{l}\text { Malignant SPLs }(\mathbf{n}=\mathbf{1 9}) \\
\text { (final diagnosis) }\end{array}$ & Total \\
\hline Benign SPLs by EUS-nCLE & 2 & 1 & 3 \\
\hline Malignant SPLs by EUS-nCLE & 1 & 18 & 19 \\
\hline Total & 3 & 19 & 22 \\
\hline
\end{tabular}

Table 3 Comparison between diagnoses of SPLs by EUS-nCLE versus final diagnostic criteria.

SPLs, solid pancreatic lesions; EUS-nCLE, endoscopic ultrasound-guided needle-based confocal laser endomicroscopy.

Eighteen patients with final diagnoses of malignant SPLs were correctly diagnosed by EUS-nCLE. One in three patients with benign SPLs were falsely diagnosed as having malignant SPLs according to the EUS-nCLE criteria. This patient had a solid pancreatic lesion due to recent acute pancreatitis. Another patient with a neuroendocrine tumor was falsely diagnosed as having benign SPLs based on the EUS-nCLE criteria.

\begin{tabular}{|l|c|}
\hline Diagnosis & Number of patients $(\mathbf{n = 2 2 )}$ \\
\hline Adenocarcinoma & 14 \\
\hline Neuroendocrine tumors & 1 \\
\hline Metastatic cancer from a salivary gland & 1 \\
\hline Lymphoma & 1 \\
\hline Malignant pancreatic lesions (unknown cell type) & 2 \\
\hline Mass-forming chronic pancreatitis & 2 \\
\hline Inflammatory mass from recent acute pancreatitis & 1 \\
\hline
\end{tabular}

Table 4 Numbers of patients with each diagnosis.

Table 5 Description of the 22 patients on a case by case basis.

\begin{tabular}{|c|c|c|c|c|c|c|c|c|}
\hline Case & Age, years & Sex & nCLE criteria & nCLE time, s & Location & Diameter, mm & nCLE diagnosis & Final diagnosis \\
\hline 1 & 46 & M & 1,2 & 1920 & Body & 45 & Benign & Benign \\
\hline 2 & 59 & M & 2,3 & 1200 & Head & 31 & Malignant & Malignant \\
\hline 3 & 66 & M & 2,3 & 550 & Head & 40 & Malignant & Malignant \\
\hline 4 & 85 & M & 3,5 & 600 & Head & 40 & Malignant & Malignant \\
\hline 5 & 66 & M & 3 & 900 & Head & 32 & Malignant & Malignant \\
\hline 6 & 49 & $\mathrm{~F}$ & 2,3 & 810 & Head & 10 & Malignant & Malignant \\
\hline 7 & 64 & $\mathrm{~F}$ & 3,5 & 240 & Tail & 44 & Malignant & Malignant \\
\hline 8 & 50 & $M$ & $2,3,5$ & 560 & Body & 30 & Malignant & Malignant \\
\hline 9 & 56 & M & 3 & 580 & Head & 31 & Malignant & Benign \\
\hline 10 & 36 & $M$ & 1,4 & 290 & Head & 26 & Benign & Benign \\
\hline 11 & 65 & M & 2,3 & 180 & Head & 58 & Malignant & Malignant \\
\hline 12 & 85 & $\mathrm{~F}$ & 3 & 500 & Head & 23 & Malignant & Malignant \\
\hline 13 & 81 & M & 2,3 & 330 & Body & 43 & Malignant & Malignant \\
\hline 14 & 66 & M & 3,5 & 560 & Head & 37 & Malignant & Malignant \\
\hline 15 & 44 & $\mathrm{~F}$ & 2,3 & 510 & Head & 24 & Malignant & Malignant \\
\hline 16 & 59 & $\mathrm{~F}$ & 2,3 & 480 & Body & 40 & Malignant & Malignant \\
\hline 17 & 75 & M & 2,3 & 230 & Head & 40 & Malignant & Malignant \\
\hline 18 & 72 & $\mathrm{~F}$ & $2,3,5$ & 100 & Head & 57 & Malignant & Malignant \\
\hline 19 & 43 & M & $1,2,4$ & 60 & Head & 30 & Benign & Malignant \\
\hline 20 & 72 & $\mathrm{~F}$ & 2,3 & 240 & Body & 40 & Malignant & Malignant \\
\hline 21 & 75 & $M$ & 3 & 80 & Head & 30 & Malignant & Malignant \\
\hline 22 & 66 & $\mathrm{~F}$ & 3 & 120 & Body & 41 & Malignant & Malignant \\
\hline
\end{tabular}

EUS-nCLE, endoscopic ultrasound-guided needle-based confocal laser endomicroscopy.

Information includes age, sex, criteria for EUS-nCLE (1, fine white fibrous bands; 2 , small black cell movement; 3, dark clumping > 40 $\mu$ m; 4, normal acinar cells; 5, dilated vessels), time for EUS-nCLE procedure, location and diameter of lesions, nCLE diagnosis and final diagnosis.

pain from a pseudocyst with main pancreatic duct obstruction at the head of the pancreas and portal vein invasion was falsely diagnosed as a benign SPL by EUS-nCLE findings. In the latter case, the nCLE finding showed only fibrous bands. Consequently, the patient developed liver metastasis during follow-up. Subsequent pathological results of EUS-FNA from the pancreatic mass confirmed the pancreatic neuroendocrine tumor. In this case, we hypothesized that the EUS-nCLE needle punctured into the inflammatory area rather than the pancreatic neuroendocrine tumor. This resulted in a false negative EUS-nCLE diagnosis.

At the end of the study, all nCLE images were presented in off-line mode to three experienced endoscopists (P.K., R.P., and P.P) who were blinded to all clinical and other related information. Diag- noses were individually made and calculated for an inter-observer agreement value. As a result, the inter-observer agreement for diagnosing SPLs with nCLE findings was almost perfect (Kappa 0.82).

A procedure-related adverse event occurred in one patient, a 66-year-old man who underwent successful EUS-nCLE and EUS-FNA procedures. During the procedure, multiple collateral vessels surrounding the SPL were noted. After the procedure, significant bloody content was noted endoscopically during withdrawal of the echoendoscope. This patient's hematocrit level dropped by $3 \%$, but no blood transfusion was required. The patient was admitted to the hospital for 2 days for close observation and was discharged uneventfully. 


\section{Discussion}

$\nabla$

Confocal laser endomicroscopy (CLE) is a novel technology providing real-time magnified endoscopic images at the cellular level at 1000-fold magnification. CLE has been used in several studies to facilitate a real-time diagnosis of mucosal diseases including Barrett's esophagus, gastric metaplasia, and colonic lesions [7-10]. A recent meta-analysis of 11 studies of CLE performed to differentiate neoplastic and non-neoplastic lesions reported a sensitivity, specificity, and negative predictive value (NPV) of $94 \%, 90 \%$, and $95 \%$, respectively [11]. Recently, needle-based confocal laser endomicroscopy (nCLE) has been introduced [12, 13]. The diameter of the nCLE miniprobe is less than $1 \mathrm{~mm}$, thus, the miniprobe can be inserted through a 19-gauge EUS-FNA needle [14]. Consequently, endoscopic ultrasound guided nCLE (EUSnCLE) was first used to demonstrate its feasibility by Konda et al. in 2011 [5]. Subsequently, a few studies used it for evaluation of cystic and solid pancreatic lesions [4,15-18]. At the time of study initiation, EUS-nCLE signs in SPLs had been reported in a multi-center trial, the Clinical Evaluation of Needle-Based Confocal LASER Endomicroscopy (nCLE) for the Diagnosis of Pancreatic Masses (CONTACT) study in abstract form [4]. In 17 from 18 malignant SPLs, Giovannini et al. described two findings of "dark cell aggregates with pseudo-glandular aspects, and straight hyperdense elements more or less thick corresponding to tumoral fibrosis. This last element was preposterous in the fibrous stroma tumor". The authors also stated that "both signs were absent in the tumors with acini cells and endocrine tumor" and "normal pancreas shows an appearance of coffee beans corresponding to acinis". These findings were determined by the consensus of four investigators and one anatomopathologist who had seen all related clinical and available pathological information [4]. However, this current study is the first pilot one to evaluate its efficacy with a prospective study design.

EUS-FNA is currently the gold standard for diagnosing SPLs, with sensitivity ranging from $86.8 \%$ to $91.0 \%$ and specificity from $95.0 \%$ to $95.8 \%[1,19]$. However, this is a demanding technique, as its efficacy is associated with several factors. The most important factor appears to be the presence of an onsite pathologist $[20,21]$; unfortunately, this immediate evaluation requires time for cytology staining and interpretation and this in turn can result in a longer time for the procedure. In addition, the optimal number of EUS-FNA passes needed to obtain a correct diagnosis of pancreatic masses is 7 [22]. In the present study, EUS-nCLE required $1-2$ passes to diagnose SPLs, with a median procedure duration of only approximately 8 minutes. This result supports the use of EUS-nCLE in clinical practice to potentially reduce procedure time and without the need for an onsite pathologist to rapidly interpret the specimen.

Regarding EUS-nCLE findings from SPLs, in this current study, during the study time, we continuously gained more experience and became more familiar with the technique on a case by case basis by comparing our findings with the final diagnoses. At the end of the study, we collected all EUS-nCLE images from SPLs and classified these findings into groups of malignant and benign SPLs. Although a head-to-head comparison with pathology was not performed in our study, malignant cells were detected in the same procedure as EUS-nCLE in the majority of malignant SPLS (13/16 cases). Before the present study, a recent study by Giovannini et al., the CONTACT study, had addressed this issue as discussed above [4]. The study was an open-label one; all participants knew all of the related information before correlating the nCLE signs with the final diagnoses. No sensitivity, specificity, or accuracy rates were reported. Another small case series of four SPLs from Giovannini et al. reported large dark clumps which were claimed to correspond with humps of malignant cells [17]. Our study is the first that systematically evaluated the efficacy of EUS-nCLE for distinguishing between benign and malignant SPLs in a blinded manner. In comparison, the strengths of both studies were different; the CONTACT study correlated EUS-nCLE findings with histopathological results and these were reviewed by experts whereas this present study interpreted images in a systematically prospective blinded fashion.

Both studies showed a similar criterion for malignant SPLs by describing them as dark clumps whereas the benign SPL was described as "find white fibrous bands and normal acinar cells in benign SPLs" in our study, and described as "normal pancreas shows an appearance of coffee beans corresponding to acinis" in the CONTACT study. In this current study, the inter-observer agreement for diagnosing SPLs with nCLE findings was almost perfect. This suggests that the criteria used in the present study are simple and likely applicable to daily clinical practice. In summary, the CONTACT study and the present study yielded some specific findings from nCLE imaging that can reliably distinguish between benign and malignant SPLs. Nevertheless, based on the results from these two studies and another small case series, a future study comparing findings from EUS-nCLE and histopathology in a prospective systematically blinded design is warranted. No procedure related adverse events were reported in our series. In fact, based on available data, no adverse events associated with EUS-nCLE for SPLs have been reported among 20 patients from the two available studies [4,5]. Unlike EUS-nCLE in cystic lesions, EUS-nCLE for SPLs had a very low rate of adverse events. Konda et al. reported two adverse events of acute pancreatitis from 16 EUS-nCLE procedures (12.5\%) for pancreatic cysts. In another multi-center study, the adverse event rate of EUS-nCLE was reported as $9 \%$ when the procedure was performed in pancreatic cysts [16].

The distinguishable features associated with nCLE imaging could encourage other researchers and endosonographers to extend this study. The present study has several limitations, including a small number of patients, the lack of available surgical pathology for all patients, no direct correlation between the nCLE findings and histopathology, no data on intra-observer variations, limited varieties of SPL types, study cohort under-represented benign disease, and a single-center design. Larger studies with a similar design are strongly recommended to include all varieties of SPLS and to correlate EUS-nCLE findings with histopathology before EUS-nCLE can become a standard investigational tool for SPLs. Despite these limitations, the current study successfully demonstrated the feasibility of the EUS-nCLE technique for the diagnosis of SPLs. The promising results of this study warrant a further study with a larger population in the future.

In conclusion, EUS-nCLE can be safely performed on SPLS, as demonstrated by the present study. The initial results of this study indicate that this approach is a promising ancillary technique that may be used to distinguish between benign and malignant SPLs. Good inter-observer agreement in this present study suggested that the nCLE criteria used are simple and likely applicable in daily practice. The real-time histology can potentially save time and reduce the use of unnecessary resources required for the EUS-FNA procedure; however, the current price of the nCLE probe is probably too high to be a cost-effective method. Larger systematic studies are needed to address other topics, such as in- 
cluding all varieties of SPLs, evaluating intra- and inter-observer variations, correlating the results with histopathology, and establishing the standard criteria.

\section{Competing interests: None}

\section{Institutions}

${ }^{1}$ Gastrointestinal Endoscopy Excellent Center, Department of Medicine, Faculty of Medicine, Chulalongkorn University and King Chulalongkorn Memorial Hospital, Bangkok, Thailand

2 Department of Pathology, Faculty of Medicine, Chulalongkorn University and King Chulalongkorn Memorial Hospital, Bangkok, Thailand

${ }^{3}$ Department of Surgery, Faculty of Medicine, Chulalongkorn University and King Chulalongkorn Memorial Hospital, Bangkok, Thailand

\section{Acknowledgement}

This research was funded by the Ratchadapiseksompoch Endowment Fund (2015), Chulalongkorn University (CU-58-001-HR).

\section{References}

1 Hewitt MJ, McPhail MJ, Possamai L et al. EUS-guided FNA for diagnosis of solid pancreatic neoplasms: a meta-analysis. Gastrointest Endosc 2012; 75: 319-331

2 Eloubeidi MA, Varadarajulu S, Desai S et al. A prospective evaluation of an algorithm incorporating routine preoperative endoscopic ultrasound-guided fine needle aspiration in suspected pancreatic cancer. J Gastrointest Surg 2007; 11: 813-819

3 Yusuf TE, Ho S, Pavey DA et al. Retrospective analysis of the utility of endoscopic ultrasound-guided fine-needle aspiration (EUS-FNA) in pancreatic masses, using a 22-gauge or 25-gauge needle system: a multicenter experience. Endoscopy 2009; 41: 445-448

4 Giovannini M, Caillol F, Bories E et al. Mo1557 Clinical evaluation of needle-based confocal LASER endomicroscopy ( $\mathrm{nCLE}$ ) for the diagnosis of pancreatic masses (contact study). Gastrointest Endosc 2013; 77: AB425

5 Konda VJ, Aslanian HR, Wallace MB et al. First assessment of needlebased confocal laser endomicroscopy during EUS-FNA procedures of the pancreas (with videos). Gastrointest Endosc 2011; 74: 1049-1060

6 Adler DG, Jacobson BC, Davila RE et al. ASGE guideline: complications of EUS. Gastrointest Endosc 2005; 61: 8-12

7 Neumann $H$, Grauer $M$, Vieth $M$ et al. In vivo diagnosis of lymphocytic colitis by confocal laser endomicroscopy. Gut 2013; 62: 333 - 334

8 Neumann H, Vieth M, Atreya R et al. Assessment of Crohn's disease activity by confocal laser endomicroscopy. Inflamm Bowel Dis 2012; 18: $2261-2269$
9 Pittayanon R, Rerknimitr R, Wisedopas $N$ et al. Flexible spectral imaging color enhancement plus probe-based confocal laser endomicroscopy for gastric intestinal metaplasia detection. J Gastroenterol Hepatol 2013; 28: 1004-1009

10 Neumann $H$, Vieth $M$, Atreya $R$ et al. First description of eosinophilic esophagitis using confocal laser endomicroscopy (with video). Endoscopy 2011; 43: UCTN E66

11 Wanders LK, East JE, Uitentuis SE et al. Diagnostic performance of narrowed spectrum endoscopy, autofluorescence imaging, and confocal laser endomicroscopy for optical diagnosis of colonic polyps: a metaanalysis. Lancet Oncol 2013; 14: 1337 - 1347

12 Neumann $H$, Kiesslich R, Wallace MB et al. Confocal laser endomicroscopy: technical advances and clinical applications. Gastroenterology 2010; 139: 388 - 392, 392.e1-2

13 Becker V, Wallace MB, Fockens $P$ et al. Needle-based confocal endomicroscopy for in vivo histology of intra-abdominal organs: first results in a porcine model (with videos). Gastrointest Endosc 2010; 71: $1260-1266$

14 Mennone A, Nathanson MH. Needle-based confocal laser endomicroscopy to assess liver histology in vivo. Gastrointest Endosc 2011; 73: $338-344$

15 Nakai Y, Iwashita T, Park DH et al. Diagnosis of pancreatic cysts: EUSguided, through-the-needle confocal laser-induced endomicroscopy and cystoscopy trial: DETECT study. Gastrointest Endosc 2015; 81 $1204-1214$

16 Konda VJ, Meining A, Jamil LH et al. A pilot study of in vivo identification of pancreatic cystic neoplasms with needle-based confocal laser endomicroscopy under endosonographic guidance. Endoscopy 2013; 45: $1006-1013$

17 Giovannini M, Caillol F, Poizat F et al. Feasibility of intratumoral confocal microscopy under endoscopic ultrasound guidance. Endosc Ultrasound 2012; 1 : 80-83

18 Napoleon B, Lemaistre AI, Pujol B et al. A novel approach to the diagnosis of pancreatic serous cystadenoma: needle-based confocal laser endomicroscopy. Endoscopy 2015; 47: 26 - 32

19 Puli SR, Bechtold ML, Buxbaum JL et al. How good is endoscopic ultrasound-guided fine-needle aspiration in diagnosing the correct etiology for a solid pancreatic mass? A meta-analysis and systematic review Pancreas 2013; 42: 20-26

20 Hebert-Magee S, Bae S, Varadarajulu $S$ et al. The presence of a cytopathologist increases the diagnostic accuracy of endoscopic ultrasoundguided fine needle aspiration cytology for pancreatic adenocarcinoma: a meta-analysis. Cytopathology 2013; 24: 159-171

21 Schmidt RL, Witt BL, Matynia AP et al. Rapid on-site evaluation increases endoscopic ultrasound-guided fine-needle aspiration adequacy for pancreatic lesions. Dig Dis Sci 2013; 58: $872-882$

22 LeBlanc JK, Ciaccia D, Al-Assi MT et al. Optimal number of EUS-guided fine needle passes needed to obtain a correct diagnosis. Gastrointest Endosc 2004; 59: 475-481 